\section{Consumo de alcohol en la edad mediana y deterioro de la capacidad cognoscitiva en edad avanzada}

La posible relación del consumo de alcohol con el deterioro cognoscitivo y la demencia ha sido por mucho tiempo tema de interés e investigación, pero difícil de dilucidar. Algunos estudios parecen rechazar la asociación del alcohol con trastornos como la enfermedad de Alzheimer, mientras que otros indican un vínculo entre el consumo abundante de alcohol y un aumento del riesgo de demencia. Se ha propuesto que existe una relación en forma de J o de U entre el consumo de alcohol y las alteraciones cognoscitivas o la demencia; es decir, que beber de forma leve a moderada puede ejercer un efecto protector comparado con la abstención estricta o la bebida desenfrenada. Es posible que el alelo e4 de la apolipoproteína modifique los efectos del alcohol sobre el riesgo de padecer alguna disfunción cognoscitiva o demencia. Ese alelo es el único factor de riesgo genético de la demencia cuyo alcance se ha determinado en la población general.

Los autores de un estudio sobre este tema llevado a cabo en Finlandia aducen que muchas de las investigaciones anteriores adolecen de fallas que les restan valor a los resultados. A pesar de que el deterioro cognoscitivo leve es susceptible de identificación clínica y conlleva un alto riesgo de progresar a la demencia, en algunos estudios el deterioro se midió en función del desempeño de los participantes en pruebas neuropsicológicas. Los resultados no se validaron con parámetros clínicos ni diagnósticos. Además, la mayor parte fueron estudios transversales o de cohortes de edad avanzada con un tiempo de seguimiento limitado, lo que puede producir sesgos debidos a demencia subclínica y otros factores. Los investigadores finlandeses opinan que para aclarar la función del alcohol en la evolución de la demencia es necesario llevar a cabo estudios prospectivos con datos del consumo anterior de alcohol y un seguimiento a largo plazo. Considerando también que los factores de riesgo en la edad mediana contribuyen a la aparición de procesos patológicos que afectan a la capacidad cognoscitiva, se propusieron averiguar si el consumo de alcohol durante esa etapa de la vida puede influir en el riesgo posterior de demencia. Decidieron asimismo investigar si el alelo e4 de la apolipoproteína influye de algún modo en la relación de ambos factores.
En 1998 se seleccionó una muestra aleatoria final de 1018 personas de la población que habían participado en un estudio sobre factores de riesgo cardiovascular, envejecimiento y demencia durante el decenio de los setenta. En las 632 mujeres y 386 hombres incluidos, la media de edad en el antiguo estudio había sido 48,3 y en el nuevo, 71,4 años. El estudio de base contenía las historias clínicas y análisis de los genotipos E de la apolipoproteína de los participantes, así como cuestionarios en los que habían consignado sus hábitos de bebida ( $30 \%$ no bebedores, $40 \%$ bebedores esporádicos y $30 \%$ bebedores asiduos). La función cognoscitiva se midió por medio de un protocolo basado en los criterios establecidos en el Centro de Investigación sobre la Enfermedad de Alzheimer de la Clínica Mayo. Los diagnósticos de demencia y enfermedad de $\mathrm{Alz}$ heimer se fundamentaron, respectivamente, en el Manual diagnóstico y estadístico de trastornos mentales (4a . ed.) y los lineamientos del Instituto Nacional de Trastornos Neurológicos y de la Comunicación y Apoplejía, y la Asociación de la Enfermedad de Alzheimer y Trastornos Relacionados.

En $61(5,8 \%)$ de los participantes se detectó deterioro cognoscitivo leve y en $48(4,6 \%)$, demencia. Entre estos últimos, 37 (77\%) padecían enfermedad de Alzheimer. Los que tuvieron resultados negativos en todas las pruebas pasaron a formar el grupo de referencia o testigo. Los datos obtenidos se examinaron mediante rigurosos sistemas de análisis estadístico teniendo en cuenta factores como edad, sexo, escolaridad, tiempo de seguimiento, hábito de fumar, concentración de colesterol y otras variables individuales. He aquí algunos de los resultados: El consumo de alcohol durante la edad mediana se asoció al riesgo (en forma de U) de padecer deterioro cognoscitivo leve en la edad avanzada. Ese riesgo, tanto en los no bebedores como en los bebedores frecuentes, fue más alto que en los bebedores esporádicos. Por el contrario, no se encontró ninguna relación en forma de $\mathrm{U}$ con la demencia. Sin embargo, el riesgo de demencia en la edad avanzada aumentaba con el mayor consumo de alcohol en la edad mediana en los portadores del alelo e4 de la apolipoproteína. De lo anterior se desprende que el deterioro leve de la función cognoscitiva está influida por factores ambientales como el consumo frecuente de alcohol. Por otra parte, el progreso a la demencia parece requerir una combinación de factores ambientales negativos con otros 
factores de susceptibilidad genética como la presencia del alelo e4.

En algunos estudios se propone que el alcohol puede tener algún efecto protector contra el deterioro cognoscitivo. Sobre la base del estudio descrito, la conclusión es que el consumo moderado de alcohol es solamente un indicador de un estilo de vida favorable, que es de por sí protector. En cuanto al efecto del alelo e4, es posible que los portadores tengan medios de reparación neural menos eficientes que los no portadores y son, por lo tanto, susceptibles frente a los daños que suele producir el alcohol. (Anttila T, et al. Alcohol drinking in middle age and subsequent risk of mild cognitive impairment and dementia in old age: a prospective population based study. BMJ. 2004 Sept 4(7465); 329:539 E pub 2004 Aug 10, PMID:15304383.)

\section{La atención prenatal y el bajo peso al nacer}

La atención prenatal adecuada suele ser una meta importante en cualquier programa de salud de la mujer, ya que se considera la forma más rentable de mejorar los resultados del embarazo. En el Brasil, la reforma sanitaria de los años ochenta garantizó el acceso universal gratis a todos los servicios públicos bajo el nuevo Sistema Único de Salud. En 1989 se inició un programa destinado a reducir las desigualdades de acceso a la atención de salud en las mujeres, especialmente durante la gestación. El Ministerio de Salud recomendó que toda embarazada comenzara sus visitas a un centro de salud a partir del primer trimestre de la gestación y fuera revisada por un obstetra por lo menos seis veces durante los meses siguientes. Con esta estrategia se procuraba atraer a los servicios de salud a las mujeres de escasos recursos, dada la estrecha relación entre las barreras socioeconómicas y el acceso a una atención prenatal satisfactoria. Sin embargo, se han llevado a cabo muy pocos estudios para evaluar el éxito de esas medidas.

En países en desarrollo, donde las poblaciones podrían beneficiarse más del cuidado apropiado, escasea la información sobre los efectos de la atención prenatal en el bajo peso al nacer (BPN). Es interesante que en otros sitios, principalmente en países industrializados, diversos estudios indican que el aumento de la atención prenatal no ha logrado las reducciones esperadas en el BPN. Con esta perspectiva, un grupo de investigadores brasileños se dedicó a estudiar la situación. Para sus fines, un ensayo aleatorizado controlado hubiera sido ideal, pero poco ético, ya que obligaría a establecer a un grupo no atendido. En este contexto, hay que depender de estudios observacionales, a pesar de que plantean muchos problemas metodo- lógicos de sesgo y factores de confusión. Comparar el uso adecuado con el inadecuado de los servicios tiende a subestimar las ventajas de la atención prenatal, sesgo que se trató de subsanar sopesando la asociación entre esa atención y el BPN. El efecto del uso adecuado de la atención prenatal en el peso del neonato se comparó con el efecto del uso inadecuado y de la total falta de atención prenatal. Los datos de estudio se tomaron de encuestas sobre dos cohortes de nacimiento en Riberão Preto, al sureste del país: una realizada de 1978 a 1979 con 6750 pares de madres y niños, antes del inicio del programa mencionado, y otra de 1994 con 2846 pares, cinco años después de haberse puesto en marcha la estrategia. Las variables maternas independientes fueron edad, escolaridad, situación marital, número de partos, tipo de seguro de enfermedad y hábito de fumar.

Mientras que el uso de la atención prenatal mejoró en el período estudiado, de 39,4\% en 19781979 a $64,0 \%$ en 1994, el BPN aumentó de 7,2\% a $10,7 \%$. En ambas encuestas, las mujeres que no aprovecharon los servicios de salud tendieron a ser menores de 20 años, fumadoras, con menos de 12 años de escolaridad, al menos cuatro embarazos y seguro público. Las madres primíparas tendieron a usar mejor la oportunidad de atención. Solo las de 35 años o mayores de la primera cohorte acudieron al control prenatal menos de las veces recomendadas. Las desigualdades en la atención prenatal aumentaron de 1979 a 1994; mientras menos escolaridad, menos asistencia a los servicios de salud. En ambos grupos, las gestantes que no asistieron a sus citas prenatales mostraron un riesgo significativamente mayor de tener un hijo de peso bajo. En el grupo de 1994, si bien se mantuvo ese riesgo en las que no recibieron ninguna atención prenatal, desapareció la asociación entre el uso inadecuado de la atención prenatal y el BPN. Esto hace pensar que otros factores, ajenos al uso de la atención prenatal, han incrementado el BPN en los hijos de mujeres pudientes y bien cuidadas. La mayor difusión de estadísticas demográficas, la elección más frecuente de intervenciones obstétricas y las nuevas técnicas para conseguir el embarazo parecen asociarse a un aumento en el parto prematuro y BPN, especialmente en mujeres de clase adinerada. La atención prenatal intensa que supera el número de visitas recomendadas según la edad gestacional también se asocia a un mayor número de intervenciones y BPN. En algunos casos, el aumento de partos prematuros refleja los adelantos en medicina perinatal, pues la reducción de muertes fetales en mujeres que han recibido buena atención prenatal también reduce la asociación entre el uso de esa atención y el BPN. Además, el parto por cesárea es más común entre mujeres privilegiadas. Pueden alegarse otras 